\title{
DETERMINISTIC LAWS OF TIME INHOMOGENEOUS DIFFUSION PROCESSES
}

\author{
By SADAo Sato
}

It is well known that a 1-dim. diffusion process whose paths are increasing is deterministic if the process is time homogeneous. The problem we will consider is to examine whether the same assertion is hold when the process is time inhomogeneous (cf. Orey [3]) and some sufficient conditions will be given.

\section{Some Basic Properties.}

Let $\left(X_{t}\right)$ be a 1-dim. time inhomogeneous diffusion process with a transition probability $P(t, x ; u, E)$, where the term "a diffusion process" means a continuous, strong Markov and conservative process. Then the process naturally defines the so called space-time process, which is a time homogeneous diffusion process on $[0, \infty) \times R^{1}$. If we denote it as $\left(T_{t}, X_{t}\right)$, then its transition probability is given by

$$
P_{(t, x)}\left[\left(T_{s}, X_{s}\right) \in A \times E\right]=P(t, x ; t+s, E) I_{A}(t+s) .
$$

Let $\sigma_{y}$ be the hitting time of $\left(T_{t}, X_{t}\right)$ to $[0, \infty) \times[y, \infty)$, i. e.

$$
\sigma_{y}=\inf \left\{s ; X_{s} \in[y, \infty)\right\}
$$

and define a function $u(t, x)$ by

$$
u(t, x)=E_{(t, x)}\left[\exp \left(-\sigma_{y}\right)\right] .
$$

Then we have

LEMMA 1 . The $\left(Y_{s}\right)$ defined by

$$
Y_{s}=\exp \left(-s \wedge \sigma_{y}\right) u\left(t+s, X_{s \wedge \sigma_{y}}\right)
$$

is a continuous $P_{(t, x)}$-martingale.

Proof. The martingale property is obtained by the direct caluculation. The continuity is a consequence of Th. 3.1 of Watanabe [2].

Received November 2, 1978 
LEMMA 2.

(a) $u(t, x)$ is increasing in $x$.

(b) If $\left(X_{t}\right)$ is increasing, then

$$
\exp (-s) u(t+s, x) \leqq u(t, x)
$$

for every s.

Proof. A proof of (a) can be carried over following the idea of Volkonskii [4]. Let $x<z<y$ and consider the product probability space $\left(\Omega_{(t, x)} \times \Omega_{(t, z)}\right.$, $\left.P_{(t, x)} \times P_{(t, z)}\right)$. We denote the elements of $\Omega_{(t, x)}$ and $\Omega_{(t, z)}$ by $\omega$ and $\omega^{\prime}$ respectively. Let

$$
\tau\left(\omega, \omega^{\prime}\right)=\left\{\begin{array}{l}
\inf \left\{s<\sigma_{y}(\omega) \wedge \sigma_{y}\left(\omega^{\prime}\right) ; X_{s}(\omega)=X_{s}\left(\omega^{\prime}\right)\right\}, \\
\infty, \text { if }\{\cdots\} \text { is void. }
\end{array}\right.
$$

Since $\tau\left(\omega, \omega^{\prime}\right)$ is a Markov time for a fixed $\omega^{\prime}$, we have

$$
\begin{aligned}
u(t, x)= & E_{(t, x)}\left[\exp \left(-\tau\left(\omega, \omega^{\prime}\right) \wedge \sigma_{y}(\omega)\right) u\left(t+\tau\left(\omega, \omega^{\prime}\right) \wedge \sigma_{y}(\omega), X_{\tau \wedge \sigma_{y}}(\omega)\right)\right] \\
= & E_{(t, x)}\left[\tau\left(\omega, \omega^{\prime}\right)<\sigma_{y}(\omega) ; \exp (-\tau) u\left(t+\tau, X_{\tau}(\omega)\right)\right] \\
& +E_{(t, x)}\left[\tau\left(\omega, \omega^{\prime}\right)=\infty ; \exp \left(-\sigma_{y}(\omega)\right)\right]
\end{aligned}
$$

Similarly

$$
\begin{aligned}
u(t, z)= & E_{(t, z)}\left[\tau\left(\omega, \omega^{\prime}\right)<\sigma_{y}\left(\omega^{\prime}\right) ; \exp (-\tau) u\left(t+\tau, X_{\tau}\left(\omega^{\prime}\right)\right)\right] \\
& +E_{(t, z)}\left[\tau\left(\omega, \omega^{\prime}\right)=\infty ; \exp \left(-\sigma_{y}\left(\omega^{\prime}\right)\right)\right] .
\end{aligned}
$$

Take the expectations of (3) and (4) by $E_{(t, z)}$ and $E_{(t, x)}$ respectively and then take the difference of them. Noting that $\left\{\left(\omega, \omega^{\prime}\right) ; \tau\left(\omega, \omega^{\prime}\right)<\sigma_{y}(\omega)\right\}=\left\{\left(\omega, \omega^{\prime}\right)\right.$; $\left.\tau\left(\omega, \omega^{\prime}\right)<\sigma_{y}\left(\omega^{\prime}\right)\right\}, X_{\tau}(\omega)=X_{\tau}\left(\omega^{\prime}\right)$ and the Fubini theorem, we finally obtain

$$
\begin{aligned}
u(t, z)-u(t, x) & =E_{(t, x)} \times E_{(t, z)}\left[\tau\left(\omega, \omega^{\prime}\right)\right. \\
& \left.=\infty ; \exp \left(-\sigma_{y}\left(\omega^{\prime}\right)\right)-\exp \left(-\sigma_{y}(\omega)\right)\right],
\end{aligned}
$$

which proves $(\mathrm{a})$, because $\sigma_{y}\left(\omega^{\prime}\right) \leqq \sigma_{y}(\omega)$ when $\tau\left(\omega, \omega^{\prime}\right)=\infty$.

(b) is clear from the inequality;

$$
\begin{aligned}
u(t, x) & =E_{(t, x)}\left[\exp \left(-s \wedge \sigma_{y}\right) u\left(t+s, X_{s \wedge \sigma_{y}}\right)\right] \\
& \geqq \exp (-s) u(t+s, x) .
\end{aligned}
$$

\section{Deterministic Laws.}

THEOREM 1. If all the martingales of the type (2) are trivial, then the process is deterministıc.

Proof. The triviality of the martingales of the type (2) implies 


$$
\exp \left(-s \wedge \sigma_{y}\right) u\left(t+s, X_{s \wedge \sigma_{y}}\right)=u(t, x),
$$

for every s. Letting $s \uparrow \infty$, we obtain

$$
\exp \left(-\sigma_{y}\right)=u(t, x) \text {. }
$$

Thus $\sigma_{y}$ is nonrandom. Therefore $X_{t}$ must be nonrandom.

LEMMA 3. If $\left(Z_{t}\right)$ is a continuous martingale whose paths are of bounaea variation, then $\left(Z_{t}\right)$ is truvial.

For a proof, cf. Kunita and Watanabe [1], p. 213.

THEOREM 2. Let $\left(X_{s}, P_{(t, x)}\right)$ be a 1-dim. increasing time inhomogeneous diffusion process. Then one of the following conditions is sufficient for the process to be deterministic:

(i) Every $u(t, x)$ defined by (1) is increasing in $t$.

(ii) The process is time homogeneous.

(iii) For every $\sigma_{y}, w$ and $x<y$, there is a constant $K$ such that

$$
\left|u(s, z)-u\left(s, z^{\prime}\right)\right| \leqq K\left|z-z^{\prime}\right|,
$$

for every $s \in[0, w]$ and $z, z^{\prime} \in[x, y]$.

(iv) For every $\sigma_{y}, w$ and $x<y$, there is a constant $C$ such that

$$
\left|u(s, z)-u\left(s^{\prime}, z\right)\right| \leqq \mathrm{C}\left|s-s^{\prime}\right|,
$$

for every $z \in[x, y]$ and $s, s^{\prime} \in[0, w]$.

Proof. It suffices to show that each condition implies that the martingale (2) is of bounded variation. Then the conclusion follows from Lemma 3 and Theorem 1. This is trivial for (i) and (ii) by Lemma 2. Assume (iii) and take any points $\left\{s_{i}\right\}_{0 \leqq \imath \leqq n}$ such that $0 \leqq s_{0}<s_{1}<\cdots<s_{n}<w-t$. Consider the difference $\delta_{i}$;

$$
\delta_{i}=\exp \left(-s_{\imath} \wedge \sigma_{y}\right) u\left(t+s_{\imath}, X_{s_{i} \wedge \sigma_{y}}\right)-\exp \left(-s_{\imath-1} \wedge \sigma_{y}\right) u\left(t+s_{\imath-1}, X_{s_{i-1} \wedge \sigma_{y}}\right) .
$$

If $\delta_{i}$ is positive, we have by (b) of Lemma 2

$$
\begin{aligned}
\delta_{i} & \leqq \exp \left(-s_{\imath-1} \wedge \sigma_{y}\right)\left\{u\left(t+s_{\imath-1}, X_{s_{i} \wedge \sigma_{y}}\right)-u\left(t+s_{\imath-1}, X_{s_{i-1} \wedge \sigma_{y}}\right)\right\} \\
& \leqq K\left|X_{s_{i} \wedge \sigma_{y}}-X_{s_{i-1} \wedge \sigma_{y}}\right| .
\end{aligned}
$$

Thus the positive variation of the martingale (2) is dominated by $K|y-x|$. Then the total variation is necessarily finite. Under the assumption (iv), consider the difference $\eta_{\imath}$;

$$
\eta_{\imath}=u\left(t+s_{\imath}, X_{s_{i} \wedge \sigma_{y}}\right)-u\left(t+s_{\imath-1}, X_{s_{i-1} \wedge \sigma_{y}}\right) .
$$

Similarly we can prove that the negative variation of the process $u\left(t+s, X_{s \wedge \sigma_{y}}\right)$ is locally finite. Therefore the martingale (2) is of bounded variation. 


\section{REMARKS}

1. Let $g$ be a continuous function from $[0, \infty)$ to $R^{1}$. Define

$$
\sigma_{g}=\inf \left\{t ; X_{t} \geqq g(t)\right\} .
$$

Our discussion can be easily extended to such hitting times and analogous results hold.

2. Let $\left(X_{t}\right)$ be an $N$-dim. diffusion process and each component increasing. It is worthwhile to see if this process is deterministic. For this problem it will be necessary to consider the continuous martingale $u\left(X_{t}\right)$ where $u$ is a bounded measurable function from $R^{N}$ to $R^{1}$. In the 1-dim. case we can easily prove that such a martingale is trivial. But it is unknown for $N \geqq 2$.

\section{REFFERENCES}

[1] H. Kunita, S. Watanabe, On square integrable martingales, Nagoya Math. J. 30 (1967), 209-245.

[2] S. WAtANABE, On discontinuous additive functionals and Lévy measures of a Markov process, Japanese J. Math. 36 (1964), 53-70.

[3] S. OREY, Diffusion on the line and additive functionals of Brownian motion, Proceedings of the conference on stochastic differential equations and applications, Acad. Press (1977), 211-230.

[4] V.A. Volkonskir, Construction of non-homogeneous Markov processes by means of a random substitution of time, Th. of Prob. and its Appl. 6 (1961), 42-51.

Tokyo Institute of Techonology 